\title{
Application of the ITMD factorization framework to calculation of cross sections for production of forward di-jets in $\mathrm{p}+\mathrm{Pb}$ collisions
}

\author{
Krzysztof Kutak \\ Institute of Nuclear Physics, Polish Academy of Sciences, \\ Radzikowskiego 152, 31-342 Kraków, Poland \\ E-mail: Krzysztof.Kutak@ifj.edu.pl
}

We report on recent study of the production of forward di-jets in proton-proton and proton-lead collisions at the Large Hadron Collider with Improved Transversal Momentum Factorization [24]. The results as compared to results obtained within High Energy Factorization show noticable effects related to detailed treatment of nonlinear effects.

XXV International Workshop on Deep-Inelastic Scattering and Related Subjects

3-7 April 2017

University of Birmingham, UK 


\section{Introduction}

Measurements of forward jet or particle production in high-energy hadronic collisions provide unique opportunities to study the QCD dynamics [1, 2, 3, 4, 5, 6] and in particular of the non-linear parton saturation regime [7, 8]. Such processes, in which, for kinematical reasons, high-momentum partons from one of the colliding hadrons scatter with small-momentum partons from the other, are called dilute-dense collisions. Indeed, the density of the large- $x$ partons in the projectile hadron is small, while the density of the small- $x$ gluons in the target hadron is large, and the former, well understood in perturbative QCD, can be used to probe the dynamics of the latter. This is true already in proton-proton collisions, although using a target nucleus does enhance the dilute-dense asymmetry of such collisions.

RHIC measurements have provided some evidence for the presence of saturation effects in the data, the most compelling of which is the successful description of forward di-hadron production $[9,10,11]$, using the most up-to-date theoretical tools available at the time in the Color Glass Condensate (CGC) framework [12,13]. In particular, this approach predicted the suppression of azimuthal correlations in $\mathrm{d}+\mathrm{Au}$ collisions compared to $\mathrm{p}+\mathrm{p}$ collisions [7], which was observed later experimentally $[14,15]$.

In this context, we shall consider forward di-jet production in proton-lead versus proton-proton collisions. In that case, it was shown in [16] that the full complexity of the CGC machinery is not needed. Indeed, for the di-hadron process at RHIC energies, no particular ordering of the momentum scales involved is assumed in CGC calculations, while at the LHC one can take advantage of the presence of final-state partons with transverse momenta much larger than the saturation scale to obtain simplifications. At present the framework does not include dynamical effects relevant at large transverse momenta, such as Sudakov logarithms [17, 18, 19, 20] or coherence in the QCD evolution of the gluon density [21, 22, 23].

The goal of this article is to report on application [24] of that new formulation, dubbed improved TMD (ITMD) factorization which is a generalization for considered process of High Energy Factorization [1, 25] and TMD factorization [26].

\section{The ITMD factorization formula for forward di-jets in dilute-dense collisions}

We consider the process of inclusive forward di-jet production in hadronic collisions

$$
p\left(p_{p}\right)+A\left(p_{A}\right) \rightarrow j_{1}\left(p_{1}\right)+j_{2}\left(p_{2}\right)+X,
$$

where the four-momenta of the projectile and the target are massless and purely longitudinal. The longitudinal momentum fractions of the incoming parton from the projectile, $x_{1}$, and the gluon from the target, $x_{2}$, can be expressed in terms of the rapidities $\left(y_{1}, y_{2}\right)$ and transverse momenta $\left(p_{t 1}, p_{t 2}\right)$ of the produced jets as

$$
x_{1}=\frac{p_{1}^{+}+p_{2}^{+}}{p_{p}^{+}}=\frac{1}{\sqrt{s}}\left(\left|p_{1 t}\right| e^{y_{1}}+\left|p_{2 t}\right| e^{y_{2}}\right), \quad x_{2}=\frac{p_{1}^{-}+p_{2}^{-}}{p_{A}^{-}}=\frac{1}{\sqrt{s}}\left(\left|p_{1 t}\right| e^{-y_{1}}+\left|p_{2 t}\right| e^{-y_{2}}\right) .
$$

By looking at jets produced in the forward direction, we effectively select those fractions to be $x_{1} \sim 1$ and $x_{2} \ll 1$. Since the target $\mathrm{A}$ is probed at low $x_{2}$, the dominant contributions come from 
the subprocesses in which the incoming parton on the target side is a gluon

$$
q g \rightarrow q g, \quad g g \rightarrow q \bar{q}, \quad g g \rightarrow g g .
$$

Moreover, the large- $x$ partons of the dilute projectile are described in terms of the usual parton distribution functions of collinear factorization $f_{a / p}\left(x_{1}, \mu^{2}\right)$ (where $\mu$ is a factorization scale) while the small- $x$ gluons of the dense target are described by TMD distributions $\Phi_{g / A}\left(x_{2}, k_{t}, \mu^{2}\right)$. Indeed, the momentum of the incoming gluon from the target is not only longitudinal but also has a nonzero transverse component of magnitude $k_{t}=\left|p_{1 t}+p_{2 t}\right|$ which leads to imbalance of transverse momentum of the produced jets: $k_{t}^{2}=\left|p_{1 t}\right|^{2}+\left|p_{2 t}\right|^{2}+2\left|p_{1 t}\right|\left|p_{2 t}\right| \cos \Delta \phi$. The validity domain of ITMD factorization is $Q_{s}\left(x_{2}\right) \ll P_{t}$ where $P_{t}$ is the hard scale of the process, related to the individual jet momenta $P_{t} \sim\left|p_{1 t}\right|,\left|p_{2 t}\right|$. By contrast, the value of $k_{t}$ can be arbitrary. The ITMD factorization formula reads [16]

$$
\frac{d \sigma^{p A \rightarrow \text { dijets }+X}}{d^{2} P_{t} d^{2} k_{t} d y_{1} d y_{2}}=\frac{\alpha_{s}^{2}\left(\mu^{2}\right)}{\left(x_{1} x_{2} s\right)^{2}} \sum_{a, c, d} \frac{x_{1} f_{a / p}\left(x_{1}, \mu^{2}\right)}{1+\delta_{c d}} \sum_{i=1}^{2} K_{a g^{*} \rightarrow c d}^{(i)}\left(P_{t}, k_{t}\right) \Phi_{a g \rightarrow c d}^{(i)}\left(x_{2}, k_{t}, \mu^{2}\right) .
$$

It involves several gluon TMDs $\Phi_{a g \rightarrow c d}^{(i)}$ (2 per channel), with different operator definitions, that are accompanied by different hard factors $K_{a g^{*} \rightarrow c d}^{(i)}$. Those where computed in [16] using either Feynman diagram techniques, or color-ordered amplitude methods. They encompass the improvement over the TMD factorization formula derived in Ref. [27] where the matrix elements were on-shell and a function of $P_{t}$ only.

We would like to point out that the ITMD factorization formula 2.4 was build in order to contain both the HEF and the TMD expressions as its limiting cases, and as such should be considered no more than an interpolating formula. We note however, that if one would be able to directly derive a factorization formula valid for $Q_{s} \ll P_{t}$ regardless of the value of $k_{t}$, any additional term compared to 2.4 should vanish in both limits $Q_{s} \sim k_{t} \ll P_{t}$ and $Q_{s} \ll k_{t} \sim P_{t}$.

\section{Numerical studies of the forward di-jet cross section}

We move now to the numerical results ${ }^{1}$ for forward di-jet production in $\mathrm{p}+\mathrm{p}$ and $\mathrm{p}+\mathrm{Pb}$ collisions at the LHC. We consider a center-of-mass energy of $8.16 \mathrm{TeV}$, and generate all our predictions with the forward region defined as the rapidity range $3.5<y<4.5$ on one side of the detector. The two hardest jets are required to lie within this region and we also impose a cut on the minimal transverse momentum of each two jets: $p_{t 0}=20 \mathrm{GeV}$. In such a setup, the cross section still may be divergent due to collinear singularities. These are cut-off by applying a jet algorithm on the final state momenta with a delta-phi-rapidity cut $R=0.5$. Finally, we require the jets to be ordered according to increasing transverse momentum, that is we have $\left|p_{t 1}\right|>\left|p_{t 2}\right|>p_{t 0}$. For the collinear parton distributions that enter the ITMD formula, we chose the general-purpose CT10 set. For the central value of the factorization and renormalization scale, we choose the average transverse momentum of the two leading jets, $\mu_{F}=\mu_{R}=\frac{1}{2}\left(\left|p_{t 1}\right|+\left|p_{t 2}\right|\right)$. We will produce error bands corresponding to the renormalization and factorization scale uncertainties by varying the central numbers from half to twice their value.

\footnotetext{
${ }^{1}$ the calculations were performed using Monte Carlo programs [28, 29]
} 

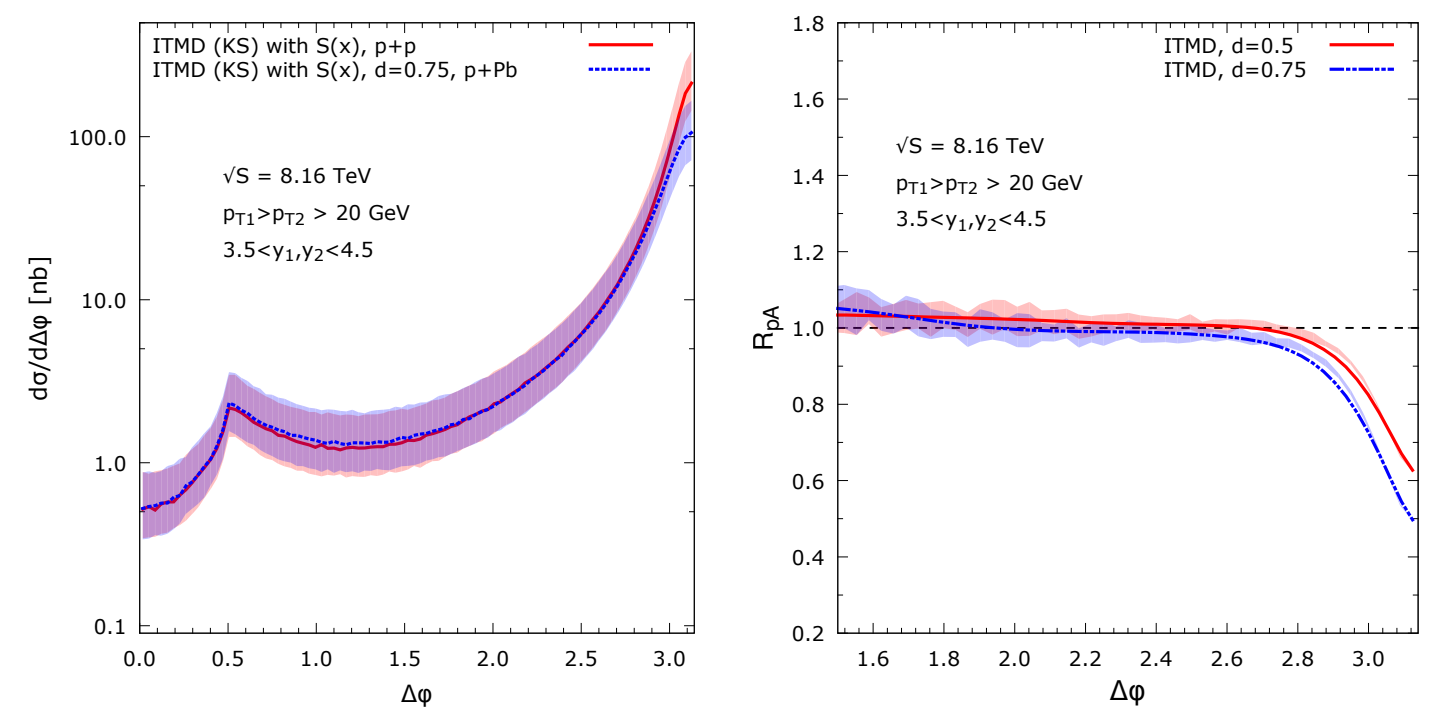

Figure 1: Left plot: differential cross section as a function of the azimuthal angle between the jets for $p+p$ and $\mathrm{p}+\mathrm{Pb}$ collisions (rescaled by the number of nucleons). The distributions are identical everywhere expect near $\Delta \phi \simeq \pi$, where saturation are the strongest. Right plot: nuclear modification factors for two values of the nuclear saturation scale, providing an uncertainty band.

For the various observables $\mathscr{O}$ shown below, we also consider the nuclear modification factors defined as

$$
R_{\mathrm{pPb}}=\frac{\frac{d \sigma^{p+P b}}{d \mathscr{O}}}{A \frac{d \sigma^{p+p}}{d \mathscr{O}}} .
$$

with $A=208$ for $\mathrm{Pb}$. In our approach, in the absence of saturation effects, or in the case in which they are equally strong in the nucleus and in the proton, this ratio is equal to unity. If, however, the non-linear evolution plays a more important role in the case of the nucleus, the $R_{\mathrm{pPb}}$ ratio will be suppressed below 1 .

We start by investigating the azimuthal correlations, with the azimuthal angle between the jets $\Delta \phi$ defined to lie within $0<\Delta \phi<\pi$.

In Fig. 1 we compare the $\Delta \Phi$ distribution in $\mathrm{p}+\mathrm{p}$ and $\mathrm{p}+\mathrm{Pb}$ collisions. After rescaling the $\mathrm{p}+\mathrm{Pb}$ cross section by the number of nucleons, we obtain identical distributions almost everywhere. It is only for nearly back-to-back jets, around $\Delta \phi \simeq \pi$, that saturation effects induce a difference. This difference is better appreciated on the nuclear modification factor, which goes from unity to 0.6, as $\Delta \phi$ varies from $\sim 2.7$ to $\pi$. Two values of the parameter $c$ have been considered, which makes up an uncertainty band that turns out to be rather small. This means that the uncertainty related to the value of the saturation scale of the lead nucleus does not strongly influence the predicted $R_{\mathrm{pPb}}$ suppression.

Finally, in Fig. 2 we display the nuclear modification factors as a function of the transverse momentum of the leading and sub-leading jet. Our conclusions are similar for these observables: the new ITMD predictions are similar to the previously obtained HEF results, due to the fact that the 

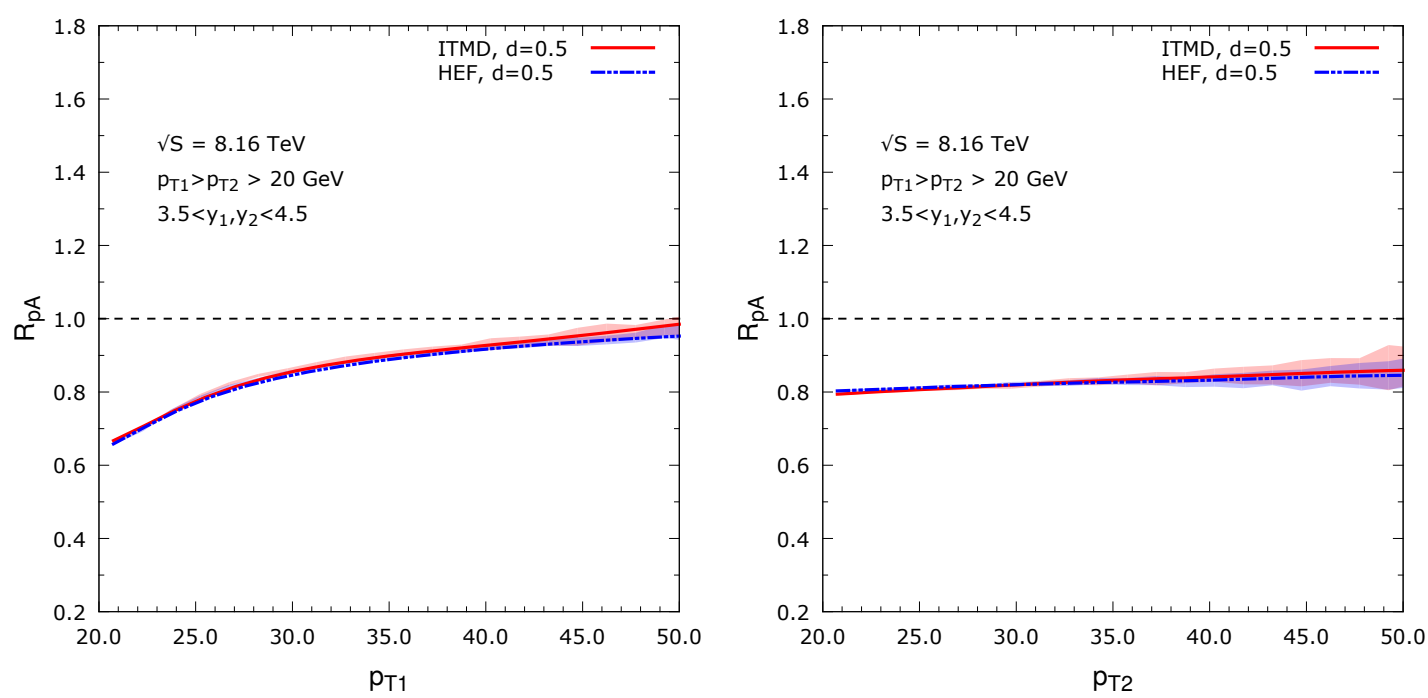

Figure 2: Nuclear modification factors as a function of the transverse momentum of the leading (left) and subleading (right) jet, comparing the new ITMD approach with previously obtained HEF results.

ITMD/HEF ratio is similar in $\mathrm{p}+\mathrm{p}$ and $\mathrm{p}+\mathrm{Pb}$ collisions. This means that the HEF framework, which is incorrect for nearly back-to-back jets - since in this formalism all the gluon TMDs are considered equal regardless of the kinematics - can nevertheless be safely used for $R_{\mathrm{pPb}}$ calculations.

\section{Conclusions}

In this paper, we reported on recent study of forward di-jet production in proton-proton and proton-lead collisions, using the small-x improved TMD factorization framework. In particular we reporten on the first numerical implementation of this formalism, and the first predictions for forward di-jets at the LHC, a process which is particularly interesting from small-x point of view. Our results for the nuclear modification factors in $\mathrm{p}+\mathrm{Pb}$ vs $\mathrm{p}+\mathrm{p}$ collisions confirm the conclusions obtained in [30] in the HEF framework, that for nearly back-to-back jets, non negligible effects of gluon saturation are to be expected as one goes from $\mathrm{p}+\mathrm{p}$ to $\mathrm{p}+\mathrm{Pb}$ collisions.

\section{Acknowledgements}

The research has been support by Narodowe Centrum Nauki with Sonata Bis grant DEC2013/10/E/ST2/00656.

\section{References}

[1] M. Deak, F. Hautmann, H. Jung and K. Kutak, JHEP 0909 (2009) 121 doi:10.1088/1126-6708/2009/09/121 [arXiv:0908.0538 [hep-ph]].

[2] M. Deak, F. Hautmann, H. Jung and K. Kutak, arXiv:0908.1870 [hep-ph].

[3] M. Deak, F. Hautmann, H. Jung and K. Kutak, arXiv:1012.6037 [hep-ph]. 
[4] M. Deak, F. Hautmann, H. Jung and K. Kutak, Eur. Phys. J. C 72 (2012) 1982 doi:10.1140/epjc/s10052-012-1982-5 [arXiv:1112.6354 [hep-ph]].

[5] M. Deak, F. Hautmann, H. Jung and K. Kutak, arXiv:1112.6386 [hep-ph].

[6] K. Kutak and S. Sapeta, Phys. Rev. D 86 (2012) 094043 doi:10.1103/PhysRevD.86.094043 [arXiv:1205.5035 [hep-ph]].

[7] C. Marquet, Nucl. Phys. A 796 (2007) 41.

[8] L. V. Gribov, E. M. Levin and M. G. Ryskin, Phys. Rept. 100 (1983) 1.

[9] J. L. Albacete and C. Marquet, Phys. Rev. Lett. 105 (2010) 162301.

[10] A. Stasto, B. -W. Xiao and F. Yuan, Phys. Lett. B 716 (2012) 430.

[11] T. Lappi and H. Mantysaari, Nucl. Phys. A 908 (2013) 51.

[12] F. Gelis, E. Iancu, J. Jalilian-Marian and R. Venugopalan, Ann. Rev. Nucl. Part. Sci. 60 (2010) 463.

[13] J. L. Albacete and C. Marquet, Prog. Part. Nucl. Phys. 76 (2014) 1.

[14] A. Adare et al. [PHENIX Collaboration], Phys. Rev. Lett. 107 (2011) 172301.

[15] E. Braidot [STAR Collaboration], arXiv:1005.2378 [hep-ph].

[16] P. Kotko, K. Kutak, C. Marquet, E. Petreska, S. Sapeta and A. van Hameren, JHEP 1509 (2015) 106

[17] A. H. Mueller, B. -W. Xiao and F. Yuan, Phys. Rev. Lett. 110 (2013) 082301.

[18] A. H. Mueller, B. -W. Xiao and F. Yuan, Phys. Rev. D 88 (2013) 114010.

[19] A. van Hameren, P. Kotko, K. Kutak and S. Sapeta, Phys. Lett. B 737 (2014) 335.

[20] K. Kutak, Phys. Rev. D 91 (2015) no.3, 034021.

[21] M. Ciafaloni, Nucl. Phys. B 296 (1988) 49.

[22] S. Catani, F. Fiorani and G. Marchesini, Nucl. Phys. B 336 (1990) 18.

[23] S. Catani, F. Fiorani and G. Marchesini, Phys. Lett. B 234 (1990) 339.

[24] A. van Hameren, P. Kotko, K. Kutak, C. Marquet, E. Petreska and S. Sapeta, JHEP 1612 (2016) 034

[25] S. Catani, M. Ciafaloni and F. Hautmann, Nucl. Phys. B 366 (1991) 135.

[26] R. Angeles-Martinez et al., Acta Phys. Polon. B 46 (2015) no.12, 2501 doi:10.5506/APhysPolB.46.2501 [arXiv:1507.05267 [hep-ph]].

[27] F. Dominguez, C. Marquet, B. W. Xiao and F. Yuan, Phys. Rev. D 83 (2011) 105005 doi:10.1103/PhysRevD.83.105005 [arXiv:1101.0715 [hep-ph]].

[28] A. van Hameren, arXiv:1611.00680 [hep-ph].

[29] P. Kotko. LxJet, the code is available at LxJet.html. http://annapurna.ifj.edu.pl/ pkotko/

[30] A. van Hameren, P. Kotko, K. Kutak, C. Marquet and S. Sapeta, Phys. Rev. D 89 (2014) no.9, 094014 doi:10.1103/PhysRevD.89.094014 [arXiv:1402.5065 [hep-ph]]. 